\title{
Histone H3K36I mutation in a metastatic histiocytic tumor of the skull and response to sarcoma chemotherapy
}

\author{
Matija Snuderl, ${ }^{1}$ Igor Dolgalev, ${ }^{2}$ Adriana Heguy, ${ }^{1}$ Michael F. Walsh, ${ }^{3}$ \\ Ryma Benayed, ${ }^{4}$ Achim A. Jungbluth, ${ }^{4}$ Marc Ladanyi, ${ }^{4}$ and Matthias A. Karajannis ${ }^{3}$ \\ ${ }^{1}$ Department of Pathology, ${ }^{2}$ Genome Technology Center, NYU Langone Medical Center, New York, New York \\ 10016, USA; ${ }^{3}$ Department of Pediatrics, ${ }^{4}$ Department of Pathology, Memorial Sloan Kettering Cancer Center, \\ New York, New York 10065, USA
}

Abstract Recurrent somatic missense mutations in histone $\mathrm{H} 3$ genes have been identified in subsets of pediatric cancers. $\mathrm{H} 3 \mathrm{~K} 36$ histone mutations have recently been recognized as oncogenic drivers in rare subsets of malignant soft tissue sarcomas but have not been reported in histiocytic neoplasms. Currently, the histological and molecular spectrum, as well as the clinical behavior of H3K36-mutant soft tissue malignancies, is largely unknown. We describe a pediatric patient with a HIST1H3B K36I-mutant histiocytic tumor arising in the skull. After the failure of upfront therapy for histiocytosis and development of widely disseminated metastatic disease, the patient had an exceptional response to empiric chemotherapy and remains in complete disease remission for more than 5 years. Our report expands the histological spectrum of H3K36M/I-mutant soft tissue malignancies to histiocytic neoplasms and indicates that multiagent sarcoma-like chemotherapy can be highly effective even in the setting of widely disseminated metastatic disease.

Corresponding author: karajanm@mskcc.org

(C) 2019 Snuderl et al. This article is distributed under the terms of the Creative Commons Attribution-NonCommercial License, which permits reuse and redistribution, except for commercial purposes, provided that the original author and source are credited.

Ontology term: neoplasm of the nervous system

Published by Cold Spring Harbor Laboratory Press

doi:10.1101/mcs.a004606

\section{INTRODUCTION}

Somatic missense mutations in histone $\mathrm{H} 3$ genes have been identified as recurrent events in a subset of pediatric high-grade gliomas, and less commonly found in soft tissue and bone tumors. Lysine 36-to-methionine (K36M) mutations in H3F3B have been found in the majority of chondroblastomas (Behjati et al. 2013), and more recently, H3 mutations at lysine 36 have also been identified in two children with undifferentiated sarcomas: a HIST1H3C K36M and a HIST1H3B K36I mutation (Lu et al. 2016). The histone 3.3 K36M mutation impairs mesenchymal differentiation and has been shown to generate undifferentiated sarcomas in vivo through epigenetic reprogramming (Fang et al. 2016; Lu et al. 2016). H3K36M/I mutations lead to the reduction of H3K36 methylation and a simultaneous genome-wide gain in H3K27 methylation, resulting in a redistribution of Polycomb repressive complex 1 (PRC1) and derepression of PRC1 target genes associated with the blocking of mesenchymal differentiation.

\section{RESULTS}

Clinical Presentation, Family History, and Pathology

A 13-yr-old African-American female, with an unremarkable past medical history except for sickle cell trait, presented with a 2-wk history of recurrent progressive headaches, nausea, 

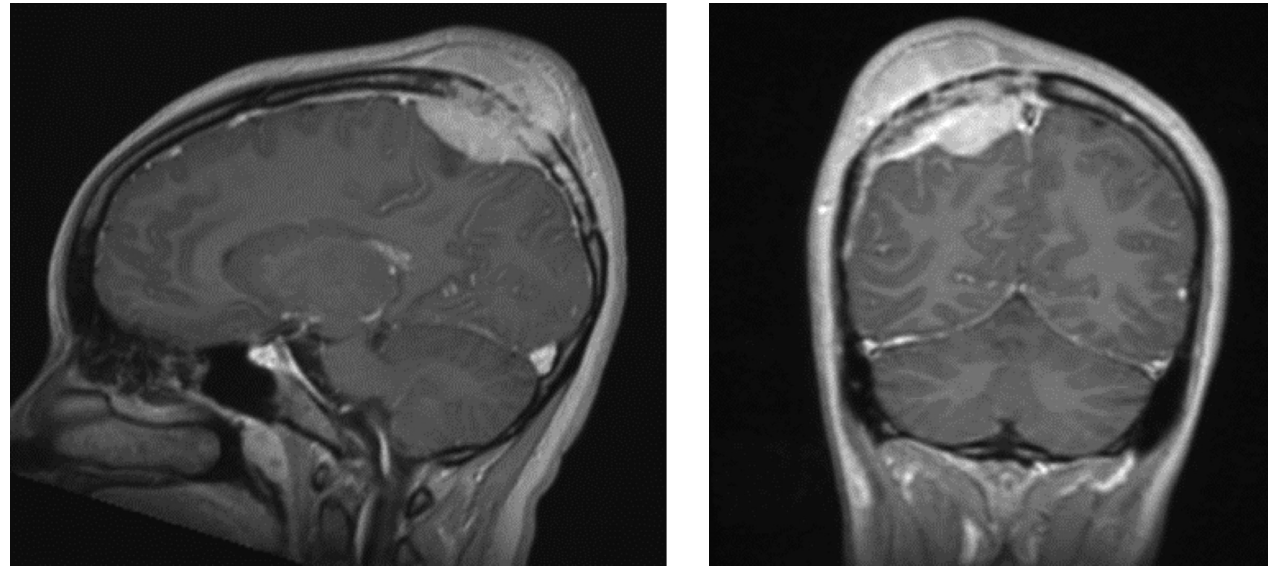

Figure 1. Initial imaging studies. (Left) Sagittal and (right) coronal contrast-enhanced, T1-weighted MR images of the head obtained at initial diagnosis, showing a homogeneously enhancing mass centered in the right parietal bone, with extracalvarial and intracranial components and associated mass effect on the parietal lobe.

and vomiting. Physical examination revealed a palpable mass involving her right parietal scalp. Family history was negative for cancer and heritable disorders.

Magnetic resonance imaging (MRI) of the head with and without contrast revealed a homogeneously enhancing mass centered in the right parietal bone, with extracranial and intracranial components and associated mass effect on the parietal lobe (Fig. 1). A diagnostic skeletal survey was negative for any additional bony lesions.

An open biopsy of the skull lesion revealed an atypical histiocytic proliferation with associated T-cell infiltrate that appeared consistent with histiocytosis, although not Langerhans cell histiocytosis (Fig. 2). The lesion was composed predominantly of histiocytes that were strongly positive for CD163 and weakly positive for CD68, but negative for S100 and CD1a. The CD45-positive lymphocytic population included a CD3-positive T-cell infiltrate. A CD20- and CD79a-positive B-cell population represented only a minority of the lesion. Hematological markers ALK-1 (anaplastic lymphoma kinase), CD15, CD30, BCL-2, BCL-6, OCT-2, and PAX-5 were negative by immunohistochemistry. The tumor was negative for EBV-encoded RNA (EBER) by in situ hybridization. Soft tissue neoplasm markers a smooth muscle actin (SMA), desmin, and caldesmon were also negative. Flow cytometric analysis with a comprehensive panel of leukemia/lymphoma markers was nondiagnostic. The Ki-67 proliferation index ranged widely between various areas of the tumor, from $<5 \%$ to $30 \%$ focally, with the marked inflammatory cell infiltrate component rendering it difficult to estimate the tumor cell proliferation rate.

Given the diagnostic uncertainty, a second, larger excisional biopsy was subsequently performed, and an atypical lymphohistiocytic lesion was again identified. Immunohistochemically, the atypical cells continued to be positive for AE1/AE3 (focal), vimentin, and $\mathrm{Ki}-67$ (with low to moderate proliferative rate similar to the original biopsy) and negative for CD31, CD4, CD163, CD68, CD34, CD33, Factor XIIla, CD15, CD30, CD21, CD20, CD3, Lysozyme, CD1a, S100, ALK, MPO, FLI-1, SMA, and EBV (EBER in situ hybridization).

In light of these findings, a diagnosis of Erdheim-Chester disease (ECD) was favored. The pathology was subsequently reviewed by several outside expert hematopathology and soft tissue pathology consultants, with diagnostic impressions including a nonneoplastic reactive process and malignant neoplasms including non-Langerhans cell histiocytosis, histiocytic sarcoma, and inflammatory fibrosarcoma. 

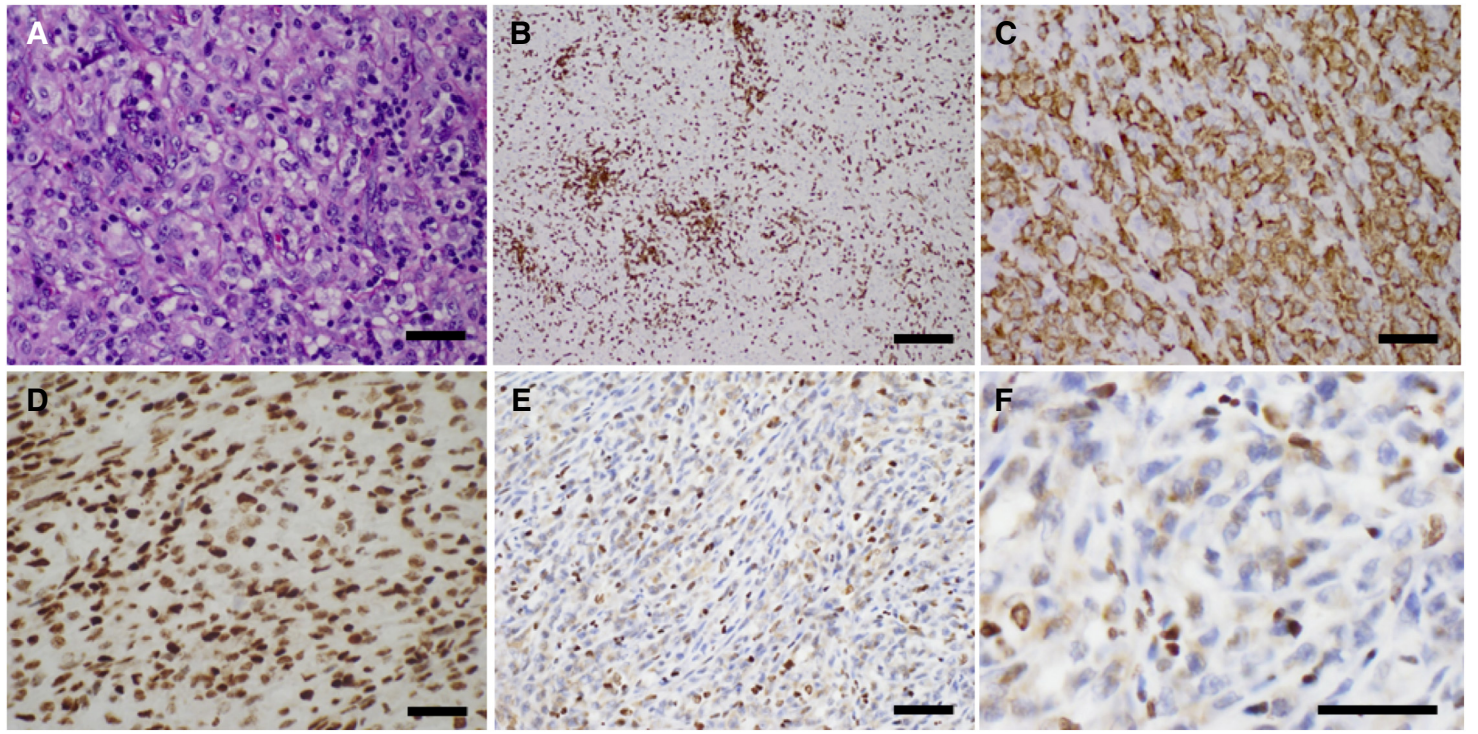

Figure 2. Tumor histology and immunohistochemistry. (A) Hematoxylin \& eosin stain showing lymphohistiocytic neoplasm with $(B)$ rich CD3-positive T-cell infiltrate and $(C)$ CD163 positivity. (D) Cells show overall preserved H3K36me3 in both tumor and inflammatory cells. $(E, F)$ In contrast, loss of H3K36me2 staining is observed in large histiocytic cells, whereas infiltrating small T cells show preserved expression. Scale bars, $100 \mu \mathrm{m}$.

\section{Genomic and Immunohistochemistry Analysis}

Whole-exome sequencing (WES) of the tumor and matched germline identified 20 somatic nonsynonymous single-nucleotide variants (SNVs) and two insertions, a frameshift insertion involving SGSM1 and a nonframe insertion involving FAM194A (Table 1). We identified a somatic mutation in HIST1H3B with an allele frequency of $20 \%$, leading to a K36I amino acid substitution (Fig. 3). No gene fusions were identified by targeted heme and solid RNA-seq fusion panels. Immunohistochemistry for histone H3K36me2 showed decreased staining in large $\mathrm{CD}_{163^{+}}$tumor cells (Fig. 2) compared to the reactive T cells' inflammatory infiltrate where $\mathrm{H} 3 \mathrm{~K} 36 \mathrm{me} 2$ expression was preserved.

\section{Treatment Outcome}

After the second biopsy, treatment for presumed ECD with oral prednisone and intravenous cladribine was initiated (Myra et al. 2004). No response was observed and the patient developed locally progressive disease within the first two cycles of therapy (8 wk). Treatment was then changed to anakinra (interleukin 1 receptor antagonist) injections 100 mg subcutaneously once daily (Aouba et al. 2010). Anakinra was discontinued after 4 wk as a result of further disease progression, including the development of tumor-associated cerebral edema and papilledema. She subsequently underwent a radical, but subtotal resection of the skull mass. Intraoperatively, extensive bony and dural tumor involvement and invasion of the pial surface were evident.

A repeat PET/CT performed after recovery from surgery revealed widely disseminated disease with new avidly metabolic lesions involving the sternum, iliac bone, sacrum, and femur in addition to residual disease at the surgical site. Subsequently, clofarabine was initiated as salvage therapy (Simko et al. 2014) at $30 \mathrm{mg} / \mathrm{m}^{2} / \mathrm{d}$ for $5 \mathrm{~d}$ every $4 \mathrm{wk}$. After two treatment cycles, the disease progressed further, with increased hypermetabolic activity at the craniotomy site and known metastatic sites, as well as the appearance of new hypermetabolic lung nodules. 
Table 1. Somatic nonsynonymous mutations identified in the tumor

\begin{tabular}{|c|c|c|c|c|c|c|c|c|c|}
\hline Gene & Chr. & $\begin{array}{l}\text { HGVS DNA } \\
\text { reference }\end{array}$ & $\begin{array}{l}\text { HGVS protein } \\
\text { reference }\end{array}$ & $\begin{array}{l}\text { Variant } \\
\text { type }\end{array}$ & Predicted effect & $\mathrm{dbSNP}$ & Genotype & $\begin{array}{l}\text { Tumor } \\
\text { depth }\end{array}$ & $\begin{array}{l}\text { VAF } \\
\text { (\%) }\end{array}$ \\
\hline ANXA9 & Chr 1 & c.C743T & p.A248V & Exonic & Nonsynonymous SNV & & Heterozygous & 314 & 10 \\
\hline FAM189B & Chr 1 & c.G446A & p.G149E & Exonic & Nonsynonymous SNV & & Heterozygous & 290 & 19 \\
\hline WRAP73 & Chr 1 & c.C319T & p.L107F & Exonic & Nonsynonymous SNV & & Heterozygous & 115 & 20 \\
\hline $\mathrm{ZCCHC} 11$ & Chr 1 & c.C4286T & p.P1429L & Exonic & Nonsynonymous SNV & & Heterozygous & 234 & 18 \\
\hline KNDC1 & Chr 10 & c.C1708T & p.R570W & Exonic & Nonsynonymous SNV & & Heterozygous & 156 & 21 \\
\hline KIRREL3 & Chr 11 & c.C1468T & p.R490W & Exonic & nonsynonymous SNV & & Heterozygous & 238 & 23 \\
\hline PTPRQ & Chr 12 & c.G4597A & p.V1533I & Exonic & Nonsynonymous SNV & & Heterozygous & 205 & 18 \\
\hline IRG1 & Chr 13 & c.G1384A & p.V462I & Exonic & Nonsynonymous SNV & & Heterozygous & 196 & 20 \\
\hline COPS2 & Chr 15 & c.G196A & p.E66K & Exonic & Nonsynonymous SNV & & Heterozygous & 374 & 16 \\
\hline CNGB1 & Chr 16 & c. G2540A & p.G847E & Exonic & Nonsynonymous SNV & & Heterozygous & 283 & 18 \\
\hline KRT39 & Chr 17 & c.T245A & p.L82O & Exonic & Nonsynonymous SNV & & Heterozygous & 268 & 18 \\
\hline$A B C A 10$ & Chr 17 & c.T6A & p.N2K & Exonic & Nonsynonymous SNV & & Heterozygous & 332 & 18 \\
\hline LOXHD1 & Chr 18 & c.G1594A & p.D532N & Exonic & Nonsynonymous SNV & & Heterozygous & 242 & 17 \\
\hline FOXD4L1 & Chr 2 & c.G61A & p.D21N & Exonic & Nonsynonymous SNV & & Heterozygous & 619 & 17 \\
\hline DNMT3B & Chr 20 & c.G389A & p.R130H & Exonic & Nonsynonymous SNV & & Heterozygous & 281 & 19 \\
\hline UBE2E2 & Chr 3 & c.A86C & p.Q29P & Exonic & Nonsynonymous SNV & & Heterozygous & 190 & 16 \\
\hline ADAMTS19 & Chr 5 & c.A2603C & p.K868T & Exonic & Nonsynonymous SNV & & Heterozygous & 245 & 21 \\
\hline AK9 & Chr 6 & c.C2594G & p.T865S & Exonic & Nonsynonymous SNV & & Heterozygous & 194 & 16 \\
\hline HIST1H3B & Chr 6 & c.A110T & p.K36I & Exonic & $\begin{array}{c}\text { Nonsynonymous } \\
\text { SNV }\end{array}$ & & Heterozygous & 138 & 20 \\
\hline PRSS16 & Chr 6 & c.G367A & p.G123S & Exonic & Nonsynonymous SNV & & Heterozygous & 157 & 27 \\
\hline SGSM1 & Chr 22 & c.1130_1131insC & p.D377fs & Exonic & Frameshift insertion & & Heterozygous & 128 & 10 \\
\hline FAM194A & Chr 3 & $\begin{array}{l}\text { c.163_164insAG } \\
\text { GAGGAGG }\end{array}$ & p.V55delinsEEEV & Exonic & $\begin{array}{l}\text { Nonframeshift } \\
\text { insertion }\end{array}$ & & Heterozygous & 145 & 23 \\
\hline
\end{tabular}

(SNV) Single-nucleotide variant.

Given the lack of response to treatment regimens for histiocytosis/ECD and clinical behavior similar to an aggressive sarcoma, empiric chemotherapy with doxorubicin 75 $\mathrm{mg} / \mathrm{m}^{2} /$ cycle and ifosfamide $9 \mathrm{~g} / \mathrm{m}^{2} /$ cycle was initiated (Patel et al. 1998). This treatment led to an exceptional response, with near-disappearance of all PET avid metastatic lesions after two cycles of therapy (Fig. 4). After two additional treatment cycles with ifosfamide, involved field external beam radiation therapy was administered to the primary site, with 5580 cGy given in 31 fractions, and two additional cycles of chemotherapy with ifosfamide and doxorubicin were administered subsequently. The patient currently remains in complete disease remission for more than 5 years.

\section{DISCUSSION}

We report a diagnostically challenging tumor with histiocytic morphology harboring a HIST1H3B K36I mutation that did not respond to multiple histiocytosis regimens but had an excellent response to a chemotherapy and radiation therapy regimen used for soft tissue sarcomas. 
TUMOR

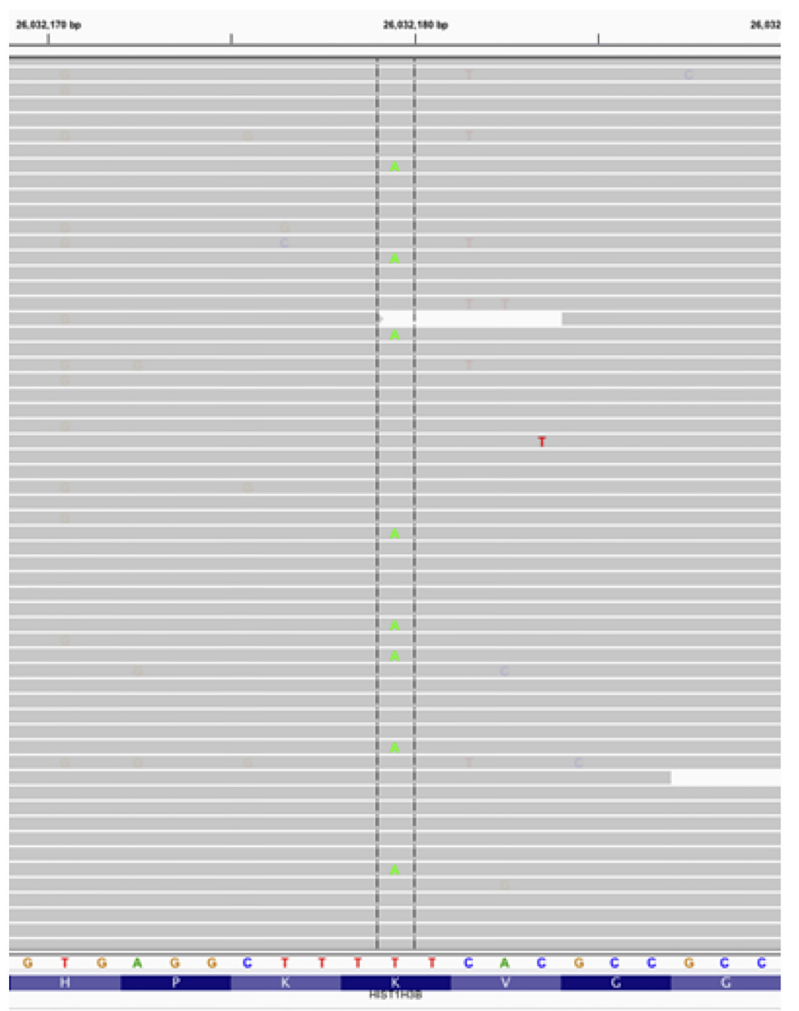

\section{GERMLINE}

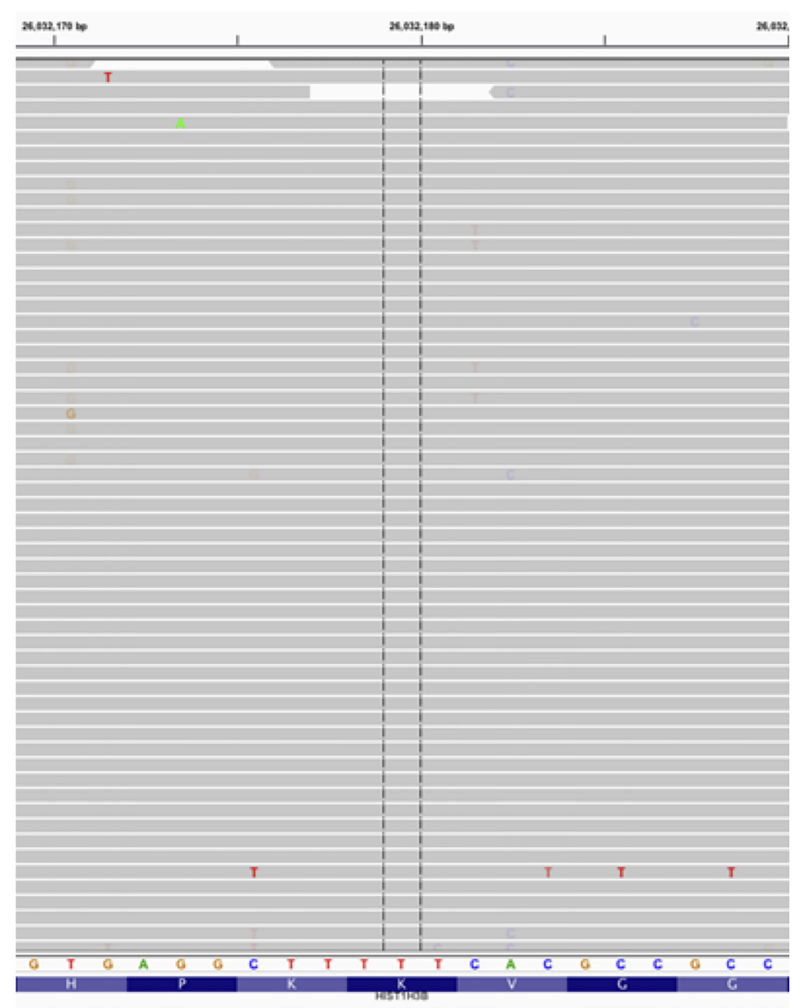

\section{HIST1H3B K36I}

Figure 3. Whole-exome sequencing. Comparison of the patient's tumor and germline DNA shows somatic HIST1H3B K36I single-nucleotide mutation with an estimated variant allele frequency of $13 \%$.

The differential diagnosis in this patient was wide-ranging and comprised both benign and malignant disease processes including non-Langerhans cell histiocytosis, histiocytic sarcoma, and inflammatory fibrosarcoma. ECD is a rare non-Langerhans cell histiocytosis with multiorgan involvement and broad clinical features (Munoz et al. 2014). Diffuse histiocytic infiltration consisting of CD68-positive and CD1a-negative lipid-laden histocytes are the traditional histological hallmarks of ECD, and a high prevalence of somatic BRAF mutations (Haroche et al. 2012) has also recently been identified in this disease. ECD was initially favored in this patient based on the presence of foamy histiocytes, rare Touton-like giant cells, factor XIII positivity, lymphocytic aggregates, fibrosis, and ultrastructural features. ECD is extremely rare in children, with few cases reported to date; and the histocytic lesions are most commonly located in the long bones of the lower extremities. ECD with cranial involvement is exceedingly rare (Jain et al. 2013), and our patient did not respond to therapies previously shown to be effective in ECD.

Histiocytic sarcoma (HS) was included in the initial differential diagnosis. This rare malignant tumor of histiocytic origin is thought to arise from the monocyte/macrophage lineage and is rarely seen in children (Takahashi and Nakamura 2013). The malignant cells in histiocytic sarcoma are typically positive for CD163, CD68, and lysozyme and negative for CD1a; and our patient's tumor also lacked characteristic histological features of histiocytic sarcoma, such as the diffuse noncohesive proliferation of large pleomorphic cells with multinucleated forms. 
COLD SPRING HARBOR Molecular Case Studies

Histone $\mathrm{H} 3 \mathrm{~K} 36 \mathrm{I}$ mutation in a histiocytic tumor

A

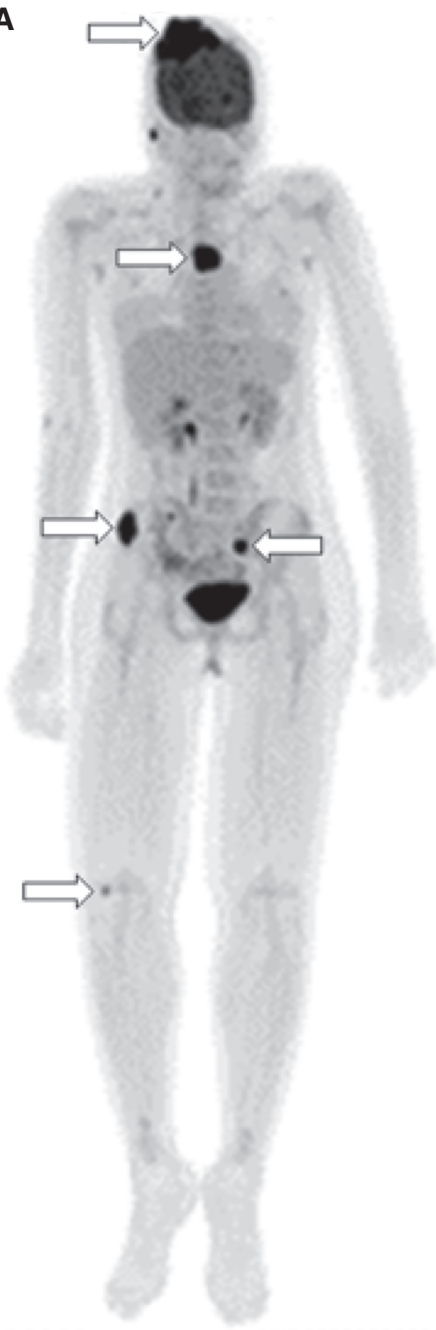

B

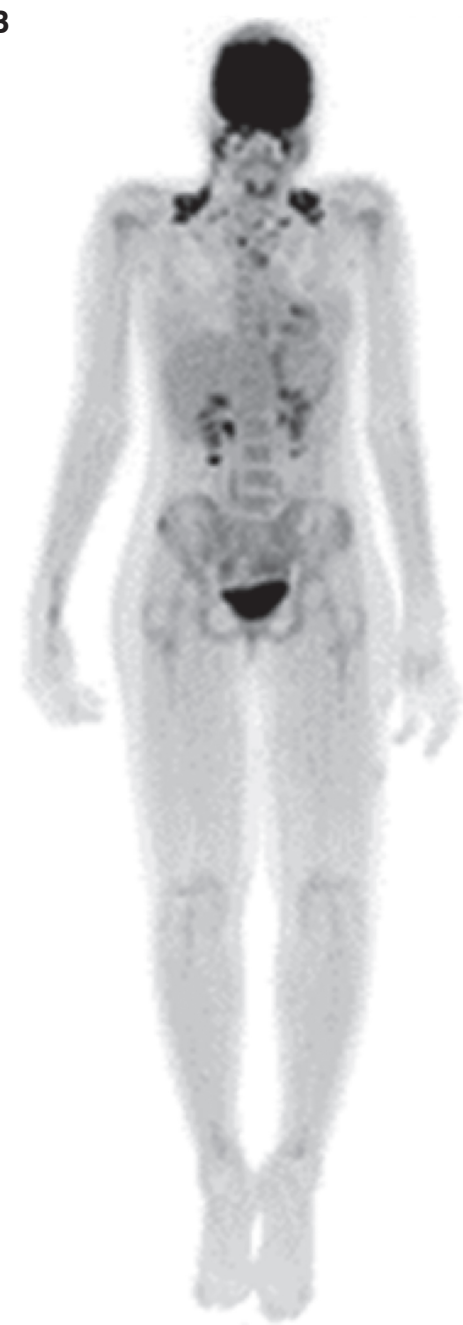

Figure 4. Disease progression and tumor response. (A) PET/CT revealing widely metastatic tumor dissemination after 6 mo of initial therapy, with hypermetabolic lesions including the primary site as well as sternum, iliac bone, sacrum, and femur (arrows). (B) Dramatic treatment response after two cycles of chemotherapy with doxorubicin and ifosfamide, with PET/CT showing near-resolution of hypermetabolic lesions.

Whole-exome sequencing of our patient's tumor revealed a single oncogenic driver alteration (i.e., a HIST1H3B K36I mutation) leading to a global reduction in H3K36me2 expression. This finding is in line with prior studies indicating that $\mathrm{H} 3 \mathrm{~K} 36 \mathrm{M} / \mathrm{I}$ mutations lead to a global reduction in H3K36me2 (Fang et al. 2016; Lu et al. 2016). To our knowledge only a single pediatric cancer patient harboring a $\mathrm{H} 3 \mathrm{~K} 36 \mathrm{l}$ has previously been described, an undifferentiated thoracic sarcoma occurring in a young child (Lu et al. 2016). No data on treatment modalities and outcome has been reported in pediatric patients with $\mathrm{H} 3 \mathrm{~K} 36 \mathrm{M} / \mathrm{I}$-mutant sarcomas. Of note, the tumor described in this report was devoid of genomic alterations in pathways frequently altered in sarcomas and associated with aggressive behavior, such as p16, p53, and RB. At present, no data exist to link the presence of a H3K36M/I mutation to responsiveness to chemotherapy. Our report expands the histological spectrum of H3K36M/I-mutant soft tissue malignancies to histiocytic neoplasms, indicating that multiagent chemotherapy can be highly effective even in the setting of widely disseminated metastatic disease. 


\section{METHODS}

We performed WES of the patient's tumor and matched germline DNA, as previously described (Snuderl et al. 2018). In brief, DNA was extracted from 10 unstained 10- $\mu \mathrm{m}$ FFPE sections using automated DNA extraction Maxwell RSC DNA FFPE (Promega). Two hundred and fifty nanograms of DNA from each sample were sheared on a Covaris instrument for $360 \mathrm{sec}$ (duty cycle-10\%; intensity-5; cycles/burst-200). Barcoded libraries were prepared using the Kapa Low-Throughput Library Preparation Kit Standard (Kapa Biosystems), amplified using the KAPA HiFi Library Amplification kit (Kapa Biosystems) (eight cycles), and quantified using Qubit Fluorimetric Quantitation (Invitrogen) and Agilent Bioanalyzer. An equimolar pool of the four barcoded libraries (300 ng each) were used as input to capture the exome using one reaction tube of the NimbleGen SeqCap EZ Human Exome Library

Table 2. The Archer custom heme and solid tumor panel were designed to target 200 and 62 specific genes, respectively, known to be recurrently involved in gene rearrangements

\begin{tabular}{|c|c|c|c|c|c|c|c|c|c|}
\hline \multicolumn{10}{|c|}{ Archer Pan Heme } \\
\hline$A B L 1^{*}$ & BTK & CHD1 & EBF1 & HOXA10 & KLF2 & MYBL1 & PAX5 & PYRY8 & sox11 \\
\hline$A B L 2$ & CALR & $\mathrm{CHIC2}$ & EIF4A1 & HOXA9 & KMT2A* & MYC & PBX1 & RAB29 & SRSF2 \\
\hline AICDA & CARD11 & CIITA & ENTPD1 & ID4 & KRAS & MYD88 & PDCD1 & RAG1 & STAT3 \\
\hline AKT3 & CBFB* & CREB3L2 & $E P O R$ & $\mathrm{IDH} 1$ & LIMD1 & MYH11 & PDCD1LG2 & RAG2 & STAT5B \\
\hline$A L K$ & $C B L$ & CREBBP & $E R G$ & IDH2 & LMO1 & NEK6 & PDGFRA & RANBP1 & STAT6 \\
\hline ASB13 & CCDC50 & CRLF2 & ETV6* & $\mid K Z F 1$ & LMO2 & NF1 & PDGFRB & RARA* & STIL \\
\hline ASXL1 & CCND1 & CSF1R & EXOC2 & IKZF2 & LRMP & NFKB1 & PHF6 & RBM15 & STRBP \\
\hline BATF3 & CCND2 & CSF3R & $E Z H 2$ & IKZF3 & LYL1 & NFKB2 & PICALM & RHOA & TAL1 \\
\hline$B A X$ & CCND3 & CTLA4 & FAM216A & IL16 & LZTS1 & NME1 & PIMI & ROSI & TCF3 \\
\hline$B C L 11 B$ & CD274 & CYB5R2 & FBXW7 & IL7R & MAL & NOTCH1 & PIM2 & RUNX1* & TFG \\
\hline$B C L 2$ & CD44 & DCK & FGFR1 & IRF4 & MALTI & NOTCH2 & PLCG1 & RUNX1T1* & $T L X 1$ \\
\hline$B C L 2 A 1$ & CD79B & DEK & FGFR2 & IRF8 & MAML3 & NPM1 & PLCG2 & S1PR2 & TLX3 \\
\hline$B C L 3$ & $C D C 25 A$ & DENND3 & FGFR3 & ITPKB & MECOM & NRAS & $P M L^{*}$ & SEMAGA & TNFRSF13B \\
\hline$B C L 6^{*}$ & CDK6 & DLEU1 & FLT3 & JAK1 & $M K L 1$ & NT5C2 & PPAT & SERPINA9 & TNFSF4 \\
\hline$B C R^{*}$ & CDKN2A & DNM2 & FOXP1 & JAK2 & MLF1 & NTRK3 & PRDM16 & SETBP1 & TP63 \\
\hline BIRC3 & CDKN2B & DNMT3A & FUT8 & JAK3 & MLLT10 & NUP214 & PRKAR2B & SETD2 & TYK2 \\
\hline BLNK & CEBPA & DNMT3B & GATA1 & KAT6A & MLLT4 & NUP98 & PTK2B & SF3B1 & U2AF1 \\
\hline$B M F$ & CEBPD & DNTT & GATA2 & KDM6A & MME & P2RY8 & PTPN1 & $\mathrm{SH} 2 \mathrm{~B} 3$ & WT1 \\
\hline BMP7 & CEBPE & DUSP22 & GLIS2 & KIAA0101 & MPL & PAG1 & PTPN11 & SH3BP5 & XPO1 \\
\hline$B R A F$ & CEBPG & E2F2 & GNAS & KIT & MUC1 & PAICS & PYCR1 & SLC29A1 & $\mathrm{ZCCHC7}$ \\
\hline \multicolumn{10}{|c|}{ Archer Solid } \\
\hline$A L K^{*}$ & $E G F R$ & $F G F R^{*}$ & JAZF1 & NCOA1 & NTRK3* & $R E T^{*}$ & TFE3* & & \\
\hline$B C O R$ & $E P C 1$ & FOSB & KIT & NCOA2 & PDGFB & $R O S 1^{*}$ & TFEB & & \\
\hline$B R A F^{*}$ & ERBB2 & FOXO1* & MAML2 & NOTCH1 & PDGFRA & RSP02 & TFG & & \\
\hline CAMTA1 & $E R G^{*}$ & FUS & MEAF6 & NOTCH2 & PHF1 & RSP03 & TMPRSS2 & & \\
\hline CCNB3 & ETV6 & GLI1 & MET & NR4A3 & PIK3CA & SS18* & USP6 & & \\
\hline $\mathrm{ClC}$ & EWSR $1 *$ & GRB7 & MGEA5 & $N R G 1$ & PLAGI & STAT6* & YWHAE & & \\
\hline COL6A3 & FGFR1 & HMGA2 & $M K L 2$ & NTRK $1 *$ & PPARG & TAF15* & & & \\
\hline DNAJB1 & FGFR2* & JAK3 & MYB & NTRK2 & PRKACA & TCF12 & & & \\
\hline
\end{tabular}

*Clinically validated genes. 
v3.0 (Roche, cat \# 06465684001), according to the manufacturer's protocol. The pooled capture library was quantified by Qubit (Invitrogen) and Bioanalyzer (Agilent) and sequenced on an Illumina HiSeq 2500 using a paired-end, 100 nt in length run mode. The coverage was normalized by HaplotypeCaller (GATK) and the variants were called independently for each sample using MuTect2 with coverage $>100 \times$ and $>10 \%$ variant allele frequency (VAF) in tumors and $<1 \%$ in the matched normal DNA. Resulting filtered variants were annotated using ANNOVAR RefSeq hg19. Synonymous and noncoding variants were excluded.

To assess for the presence of additional oncogenic drivers including gene fusions that may have been missed by WES, we analyzed the tumors using the MSK-Solid Fusion Assay, an RNA-based targeted sequencing panel that utilizes the Archer Anchored Multiplex PCR (AMPTM) technology and next-generation sequencing to detect gene fusions in hematologic and solid tumor samples (Zheng et al. 2014). The Archer custom heme and solid tumor panels were designed to target 200 and 62 specific genes, respectively, known to be recurrently involved in gene rearrangements. The complete gene list is provided in Table 2.

Briefly, RNA was extracted from tumor FFPE material followed by cDNA synthesis, cDNA end repair, dA tailing, and ligation with Illumina molecular barcode adapters. SPRI-cleaned ligated fragments are subject to two rounds of PCR amplifications using two sets of genespecific primers (GSP1 used in PCR1 and a nested GSP2 pool that is $3^{\prime}$ downstream from GSP1 and used in PCR2) and a primer complementary to the lllumina adapter. At the end of two PCR steps, the final targeted amplicons were sequenced on an Illumina MiSeq instrument $(2 \times 150 \mathrm{bp})$. The Archer analysis software V5.0 was used for data analysis, which revealed no evidence of oncogenic gene fusions in the tumor sample.

\section{ADDITIONAL INFORMATION}

\section{Data Deposition and Access}

The HIST1H3B K36I variant described in the manuscript will be deposited in ClinVar (https:// www.ncbi.nlm.nih.gov/clinvar/) under accession number SCV000993417. Raw sequencing data was not deposited because consent for public deposition of the genomic data was not granted.

\section{Ethics Statement}

The study was conducted at NYU School of Medicine and approved by the Institutional Review Board (IRB) in accordance with all local and federal regulations. The patient provided written consent for publication; however, the consent for public deposition of the genomic data was not granted.

\section{Acknowledgments}

The study was supported in part by The Making Headway Foundation and The Friedberg Charitable Foundation and through the National Institutes of Health $(\mathrm{NIH}) / \mathrm{National}$ Cancer Institute (NCl) Cancer Center Support Grant P30 CA008748 to Memorial Sloan

Competing Interest Statement

The authors have declared no competing interest.

Received July 14, 2019; accepted in revised form August 30, 2019.
Kettering Cancer Center.

\section{Author Contributions}

M.S. and M.A.K. conceived the study and wrote the manuscript. All authors acquired and analyzed the data and edited and approved the final version of the manuscript. 


\section{REFERENCES}

Aouba A, Georgin-Lavialle S, Pagnoux C, Martin Silva N, Renand A, Galateau-Salle F, Le Toquin S, Bensadoun H, Larousserie F, Silvera S, et al. 2010. Rationale and efficacy of interleukin-1 targeting in Erdheim-Chester disease. Blood 116: 4070-4076. doi:10.1182/blood-2010-04-279240

Behjati S, Tarpey PS, Presneau N, Scheipl S, Pillay N, Van Loo P, Wedge DC, Cooke SL, Gundem G, Davies H, et al. 2013. Distinct H3F3A and H3F3B driver mutations define chondroblastoma and giant cell tumor of bone. Nat Genet 45: 1479-1482. doi:10.1038/ng.2814

Fang D, Gan H, Lee JH, Han J, Wang Z, Riester SM, Jin L, Chen J, Zhou H, Wang J, et al. 2016. The histone H3.3K36M mutation reprograms the epigenome of chondroblastomas. Science 352: 1344-1348. doi:10 .1126/science.aae0065

Haroche J, Charlotte F, Arnaud L, von Deimling A, Hélias-Rodzewicz Z, Hervier B, Cohen-Aubart F, Launay D, Lesot A, Mokhtari K, et al. 2012. High prevalence of BRAF V600E mutations in Erdheim-Chester disease but not in other non-Langerhans cell histiocytoses. Blood 120: 2700-2703. doi:10.1182/blood-2012-05430140

Jain RS, Sannegowda RB, Jain R, Mathur T. 2013. Erdheim-Chester disease with isolated craniocerebral involvement. BMJ Case Rep 2013: bcr2012006823. doi:10.1136/bcr-2012-006823

Lu C, Jain SU, Hoelper D, Bechet D, Molden RC, Ran L, Murphy D, Venneti S, Hameed M, Pawel BR, et al. 2016. Histone H3K36 mutations promote sarcomagenesis through altered histone methylation landscape. Science 352: 844-849. doi:10.1126/science.aac7272

Munoz J, Janku F, Cohen PR, Kurzrock R. 2014. Erdheim-Chester disease: characteristics and management. Mayo Clin Proc 89: 985-996. doi:10.1016/j.mayocp.2014.01.023

Myra C, Sloper L, Tighe PJ, Mclntosh RS, Stevens SE, Gregson RH, Sokal M, Haynes AP, Powell RJ. 2004. Treatment of Erdheim-Chester disease with cladribine: a rational approach. Br J Ophthalmol 88: 844847. doi:10.1136/bjo.2003.035584

Patel SR, Vadhan-Raj S, Burgess MA, Plager C, Papadopolous N, Jenkins J, Benjamin RS. 1998. Results of two consecutive trials of dose-intensive chemotherapy with doxorubicin and ifosfamide in patients with sarcomas. Am J Clin Oncol 21: 317-321. doi:10.1097/00000421-199806000-00025

Simko SJ, Tran HD, Jones J, Bilgi M, Beaupin LK, Coulter D, Garrington T, McCavit TL, Moore C, RiveraOrtegón F, et al. 2014. Clofarabine salvage therapy in refractory multifocal histiocytic disorders, including Langerhans cell histiocytosis, juvenile xanthogranuloma and Rosai-Dorfman disease. Pediatr Blood Cancer 61: 479-487. doi:10.1002/pbc.24772

Snuderl M, Kannan K, Pfaff E, Wang S, Stafford JM, Serrano J, Heguy A, Ray K, Faustin A, Aminova O, et al. 2018. Recurrent homozygous deletion of DROSHA and microduplication of PDE4DIP in pineoblastoma. Nat Commun 9: 2868. doi:10.1038/s41467-018-05029-3

Takahashi E, Nakamura S. 2013. Histiocytic sarcoma: an updated literature review based on the $2008 \mathrm{WHO}$ classification. J Clin Exp Hematop 53: 1-8. doi:10.3960/jslrt.53.1

Zheng Z, Liebers M, Zhelyazkova B, Cao Y, Panditi D, Lynch KD, Chen J, Robinson HE, Shim HS, Chmielecki J, et al. 2014. Anchored multiplex PCR for targeted next-generation sequencing. Nat Med 20: 1479-1484. doi:10.1038/nm.3729 


\section{COLD SPRING HARBOR Molecular Case Studies}

\section{Histone H3K36I mutation in a metastatic histiocytic tumor of the skull and response to sarcoma chemotherapy}

Matija Snuderl, Igor Dolgalev, Adriana Heguy, et al.

Cold Spring Harb Mol Case Stud 2019, 5: a004606

Access the most recent version at doi: $10.1101 / \mathrm{mcs} . a 004606$

References

License

Email Alerting Service
This article cites 13 articles, 6 of which can be accessed free at: http://molecularcasestudies.cshlp.org/content/5/5/a004606.full.html\#ref-list-1

This article is distributed under the terms of the Creative Commons Attribution-NonCommercial License, which permits reuse and redistribution, except for commercial purposes, provided that the original author and source are credited.

Receive free email alerts when new articles cite this article - sign up in the box at the top right corner of the article or click here. 